\title{
Serological and molecular approaches in clinical diagnosis of ocular toxoplasmosis in Iran
}

\author{
Vafa Saber ${ }^{1}$, Seyyed Javad Seyyed Tabaei*2, Seyyed Ali Tabatabaei ${ }^{3}$, Mohammad Soleimani ${ }^{3}$, \\ Ali Haghighi ${ }^{2}$
}

\section{Abstract}

Background: Toxoplasma gondii ( $T$. gondii) is the most common parasite that can lead to a disease called toxoplasmosis. In this study, serological and molecular complementary tests have been conducted to detect or diagnose this parasite.

Methods: A total of 71 patients with clinical symptoms of ocular toxoplasmosis and 20 patients with other ocular infections were evaluated. Serum and buffy coat samples were collected and tested using enzyme-linked immunosorbent assay (ELISA) and nested polymerase chain reaction (nPCR) assessments. Superficial T. gondii B1 gene was evaluated in PCR. The ocular toxoplasmosis patients were followed-up 2 weeks after the first sampling and 4 weeks following the first laboratory testing. The main outcome measures were the efficiency of the diagnostic procedure and positive and negative predictive values (PPV and NPV).

Results: Overall, of the samples, $69 \%$ were PCR + , IgG+, and IgM-, and $4.2 \%$ showed PCR+, IgG + , and IgM+. In the first followup, after 2 weeks, from the 41 referred patients, $29(70 \%)$ showed PCR+, IgG+, and IgM-, which confirmed the results of the first sampling. In the second follow-up, 9 (47\%) patients were PCR+, IgG+, and IgM-. A correlation was observed between the first referral and the follow-ups. Also, from 71 patients, diagnosed clinically as ocular toxoplasmosis, the disease was confirmed in $73.2 \%$ and $26.8 \%$ of those suffering from other ocular infections. Of the 20 control group samples, 55\% showed PCR-, IgG+, and IgM-. The sensitivity, specificity, negative and positive predictive values, and negative and positive likelihoods were analyzed for $\operatorname{IgG}$ and $\operatorname{IgM}$ antibodies and for PCR using ELISA method.

Conclusion: As the ophthalmologic signs of T. gondii may be mimicked by other infections, clinical methods may be complemented by laboratory approaches for a definite diagnosis. This would assist clinicians to achieve timely diagnosis and successful therapy and to control the infection.

Keywords: Ocular toxoplasmosis, Toxoplasma gondii, Molecular approaches, Serology

\author{
Conflicts of Interest: None declared \\ Funding: None \\ *This work has been published under CC BY-NC-SA 1.0 license. \\ Copyright $($ Iran University of Medical Sciences
}

Cite this article as: Saber V, Seyyed Tabaei SJ, Tabatabaei SA, Soleimani M, Haghighi A. Serological and molecular approaches in clinical diagnosis of ocular toxoplasmosis in Iran. Med J Islam Repub Iran. 2019 (12 Aug);33:82. https://doi.org/10.47176/mjiri.33.82

\section{Introduction}

Toxoplasmosis, as one of the most common infectious diseases, results from a parasite named $T$. gondii that in-

Corresponding author: Dr Seyyed Javad Seyyed Tabaei, seyyedtabaei@sbmu.ac.ir

1. Department of Parasitology and Mycology, School of Medicine, Student Research Committee, Shahid Beheshti University of Medical Sciences, Tehran, Iran

2. Department of Parasitology and Mycology, School of Medicine, Shahid Beheshti University of Medical Sciences, Tehran, Iran

3. Farabi Hospital, Tehran University of Medical Sciences, Tehran, Iran fects a wide variety of its host's organs (1). Clinical spectrum of the infection in acquired form is not clinically

\section{$\uparrow$ What is "already known” in this topic:}

Diagnosis of toxoplasmosis in laboratory can be conducted by various tests (6), including molecular methods, histological examinations, parasite isolation following inoculation, and several serological methods. Specific DNA can be identified in the total blood samples of toxoplasma patients with ocular symptoms using the PCR method. The recognition of specific antibodies( $(\mathrm{IgG})$ in sera by ELISA method

$\rightarrow$ What this article adds:

The purpose of the method used in current study was that laboratory techniques, such as ELISA and nested PCR, can be used as complementary approaches, along with a clinical evaluation, to achieve a definite and precise diagnosis. 
significant; however, in the congenital form, it varies from asymptomatic latent infection to a fatal disease (2). Through the circulation, $T$. gondii can enter the visual system and cause irreversible optic nerve damage in neural parts of the eyes, such as choroid and retina (3).

Infection can be active or remain passive for a long time (4 months to several years). In most cases, the involvement of posterior segment of the eye or adjacent areas indicates more acute infection. Lesions of the posterior pole pose serious threats to vision throughout life. Other symptoms consist of blurred vision, photophobia, inflammation of the vitreous, formation of active and passive scars, and other clinical symptoms. In some cases, macular injury and retinal hemorrhage are observed in active and passive forms of ocular toxoplasmosis. Presence of active and inactive scars with an area of the focal necrotizing retinochoroidal lesion and focal retinochoroidal necrosis, appearing as a white-yellowish and blurred margin area with or without the old lesion, are the most obvious symptoms in ocular toxoplasmosis patients. These symptoms differ from those of other ocular diseases, which mimic some similar symptoms rather than active and passive scars. In the course of time, the lesions turn to white plaques with dark spots and distinct margins, originated from retinal pigment epithelium (RPE) (4).

The overall seroprevalence rate of toxoplasmosis among the general Iranian population has been shown to be $39.3 \%(95 \% \mathrm{CI}=33.0 \%-45.7 \%)$ in a recent systematic and meta-analysis review (5). There was no significant gender difference in the seroprevalence rates. Nevertheless, a significant linear trend of an increase in the overall prevalence by age was noted (4).

Diagnosis of toxoplasmosis in laboratory can be conducted by various tests (6), including molecular methods, histological examinations, parasite isolation following inoculation, and several serological methods. However, using only one method is not enough to distinguish ocular toxoplasmosis, particularly in the fetus and immunosuppressed patients (7) During the last years, IgG avidity test with serum, PCR with body fluids, and the immunoelectrophoresis of the umbilical cord serum have been considered as new valuable methods (8). Moreover, PCR has been used as an alternative to serology by amplification of toxoplasma DNA sequences, which are present in different clinical samples, such as amniotic fluid (9), cerebrospinal fluid (CSF) (10), tissues (11), aqueous humor (12), and human blood (13). Despite its great diagnostic power, this molecular technique is not widely employed in a clinical setting for routine diagnosis or therapeutic management of patients with toxoplasma infection (14).

The diagnosis of ocular toxoplasmosis mostly relies on clinical evaluations in Iran. However, in this study, it was aimed to demonstrate that laboratory techniques, such as ELISA and nPCR, can be used as complementary approaches, along with a clinical evaluation, to achieve a definite and precise diagnosis.

\section{Methods}

Population and sampling: A total of 71 patients (39 men and 32 women), with a mean age of 36 years (16 to 58 years), were clinically diagnosed with ocular toxoplasmosis. Moreover, 20 patients with other ocular infections and some with identical symptoms to toxoplasmosis (11 men and 9 women), with an age range of 23-41 years and average of 32 years, underwent clinical examination as a nontoxoplasma control group. For this investigation, patients from some ophthalmology centers in Tehran (Farabi, Labafinejad, and Imam Hossein Hospitals) were included. This study was approved by the review board/ethics committee of Shahid Beheshti University of Medical Sciences, Tehran, Iran (Sbmu.Rec.1392.254). An informed consent was obtained from each patient.

Moreover, the referred toxoplasmosis patients were followed-up in a 4-week period. There were 2 follow-up sessions for the patients: 2 and 4 weeks following the first laboratory evaluation. Nevertheless, the control group was not followed- up. During the course of the study, blood samples were collected 3 times and each time $5 \mathrm{~mL}$ blood was drawn from each patient and kept in 2 distinct tubes, with and without EDTA (anticoagulant).

Clinical evaluations: All patients were evaluated using direct and indirect ophthalmoscopy and funduscopy, angiography, magnetic resonance imaging (MRI) (Siemens 3T), and computed tomography (CT).

The serological and molecular test: Serological tests for IgG and IgM evaluation were performed using 2 commercial toxoplasmosis kits from Acon Company. For all patients in the first, second, and third stages, and those with other ocular diseases, the index value $>1.1$ was considered as a positive response for each IgG and IgM toxoplasma (15). The first and the second follow-ups for a group of patients with high $\operatorname{IgM}$ and $\operatorname{IgG}$ antibody titers were done within 2 weeks and 4 weeks after the first sampling, respectively.

DNA were extracted from buffy coat samples of all the ocular toxoplasmosis patients in the first referral and the first and the second follow-ups using DNG plus kit (CinnaGen Co., Iran). All samples were stored at $-20^{\circ} \mathrm{C}$ for further assessments.

B1 gene from tachyzoite surface antigen (35-foldrepetitive) was used (access no. AFyV01002650). Two pairs of internal or external primers with $579 \mathrm{bp}$ fragments and secondary or internal primer with 529 bp fragments from the first PCR product were amplified.

Nucleotide sequences of external primers are Pm1S1: F1: (5'-TGTTCTGTCCTATCGCAACG-3') and Pml/ AS1: R1: (5'-ACGGATGCAGTTCCTTTCTG-3'), and those of internal primers are Pm1/S2:F2 :(5'-TCTTCCCA GACGTGGATTTC-3') and Pm1/AS2: R2: (5'-CTCGA CAATACGCTGCTTGA-3'). PCR and Nested PCR were performed employing Taq DNA Polymerase Master Mix 2x Red (Ampliqon, Denmark kit). To perform the procedure in each reaction, $7.5 \mu \mathrm{L}$ Master Mix, $5 \mu \mathrm{L}$ distilled water, 15 picomol (Pmol) B1 primer, and $50 \mathrm{ng}$ DNA (total reaction volume of $15 \mu \mathrm{L}$ ) were used. The main mixture was prepared and divided according to the number of samples. PCR was performed in 35 cycles: the initial and secondary denaturation were done at $94^{\circ} \mathrm{C}$ for 7 minutes and 30 seconds, respectively. Annealing was performed at $60^{\circ} \mathrm{C}$ for 30 seconds. Extension and final exten- 
sion were done at $72^{\circ} \mathrm{C}$ for 30 seconds and 7 minutes, respectively $(16,17)$.

Principal outcome measure: The principal outcome measure was the efficiency of diagnostic procedure (EDP). The EDP of a test is defined as true positive tests plus true negative tests divided by the total number of tests performed. PPV and NPV were also calculated for serological and molecular evaluations. PPV is defined as true positive tests divided by all true and false positive tests. The NPV is defined as true negative tests divided by all true and false negative tests (18). For EDP, PPV, and NPV analyses, the patients were grouped according to the final diagnosis. A true positive or true negative result was considered as a test or set of tests that agreed with the final laboratory diagnosis. On the other hand, a false positive test was defined as a test that detected DNA which disagreed with the final clinical diagnosis, and a false negative was defined as a test that failed to detect DNA from the predicted etiologic agent that is detectable in the final clinical diagnosis.

\section{Results}

Various symptoms identified in the patients with ocular toxoplasmosis and other ocular problems are presented in Table 1. Most of the symptoms were observed in the optic nerve tissue, specifically in the retina, then, choroid and vitreous inflammation also occurred. In most cases, active and inactive scars and vitreous damage, in addition to other symptoms, were dominant and there were specific signs in the definite diagnosis of the infection from other ocular diseases. Posterior uveitis was observed in a large number of ocular toxoplasmosis patients. In patients with other ocular diseases (ophthalmic), as with the control group, specific symptoms were seen.

In the second stage of the disease or the first follow-up, which was done 2 weeks after the first visit, the patients had similar results in the first stage. Therefore, there was a consistent relationship between the results of the first and second stages. The third stage of the disease or the second follow-up was 4 weeks after the first stage, where the patients had the same results in the first and second stages. In this respect, there was a continuous relationship between the results of the first, second, and third stages.

Also, 2 and 4 weeks after the initial treatment, the clinical symptoms of the patients who took the prescribed drugs were still observed and the doses of the medications, drug resistance, or misdiagnosis of the cases were considered. However, a 6 -week treatment period should be considered for ocular toxoplasmosis in most clinical treatments.

The serological and molecular results of the ocular toxoplasmosis patients are presented in Tables 2 and 3. As

Table 1. Various identified symptoms of ocular toxoplasmosis and control groups

\begin{tabular}{lcc}
\hline Identified symptoms & Ocular toxoplasmosis, $\mathrm{n}=71(\%)$ & Control group, $\mathrm{n}=20(\%)$ \\
\hline blurred vision & $71(100)$ & $11(55)$ \\
intermediate uveitis & $7(9)$ & $14(70)$ \\
ophthalmia & $67(94)$ & $20(100)$ \\
posterior uveitis & $64(90)$ & - \\
inactive and old scars & $59(83)$ & - \\
chorioretinitis & $57(80)$ & - \\
Vitreous inflammation & $22(30)$ & - \\
Active scars & $12(16)$ & - \\
anterior uveitis & $8(11)$ & - \\
photophobia & $69(97)$ & - \\
cataracts & - & $9(45)$ \\
retinitis & - & $8(40)$ \\
ocular vasculitis & - & $5(25)$ \\
\hline
\end{tabular}

Table 2. Total serological and molecular results of the buffy coat and serum samples in ocular toxoplasmosis patients

\begin{tabular}{lc}
\hline Groups (number) & Results \\
\hline Control group(First step, $n=20)$ & $11(55 \%)$ IgG $^{+}$, IgM- $^{-}$PCR $^{-}$
\end{tabular}

Toxoplasmosis patients

(First step, $\mathrm{n}=71$ )

Group 1: 49 (69\%) $\mathrm{IgG}^{+}, \mathrm{IgM}^{-}, \mathrm{PCR}^{+}$

Group 2: $3(4 \%) \operatorname{IgG}^{+}, \mathrm{IgM}^{+}, \mathrm{PCR}^{+}$

Group 3: 19 (26\%) IgG', IgM-, PCR

Toxoplasmosis patients

(First follow-up, $\mathrm{n}=41$ )

$29(70 \%)$ IgG+, IgM-, PCR+

Group 1: $4(13 \%)$ decreased Ab titer

Group 2: 6 (14\%) increased Ab titer

Group 3: 19 (46\%) steady Ab titer

Toxoplasmosis patients (Second follow-up, $\mathrm{n}=19$ )

Group 1: $2(33 \%)$ increased Ab titer

$9(47 \%) \mathrm{IgG}^{+}, \mathrm{IgM}^{-}, \mathrm{PCR}^{+}$

Group 2: $2(22 \%)$ decreased Ab titer

Group 3: 4 (44\%) steady Ab titer

\begin{tabular}{|c|c|c|c|c|c|c|}
\hline Diagnostic tests & $\begin{array}{c}\text { Sensitivity } \\
(\%)\end{array}$ & $\begin{array}{c}\text { Specificity } \\
(\%)\end{array}$ & $\begin{array}{c}\text { Positive predictive } \\
\text { value }(\%)\end{array}$ & $\begin{array}{l}\text { Negative } \\
\text { predictive } \\
\text { value (\%) }\end{array}$ & $\begin{array}{c}\text { Positive likeli- } \\
\text { hood, Ratio } \\
\left(\mathrm{LR}^{+}\right)\end{array}$ & $\begin{array}{c}\text { Negative } \\
\text { likelihood, Ratio } \\
\left(\mathrm{LR}^{-}\right)\end{array}$ \\
\hline ELISA-IgG & 73 & 45 & 82 & 32 & 1.32 & 0.6 \\
\hline ELISA-IgM & 4.2 & 100 & 100 & 22.2 & - & 0.9 \\
\hline n-PCR & 47 & 100 & 100 & 35 & - & 0.53 \\
\hline
\end{tabular}




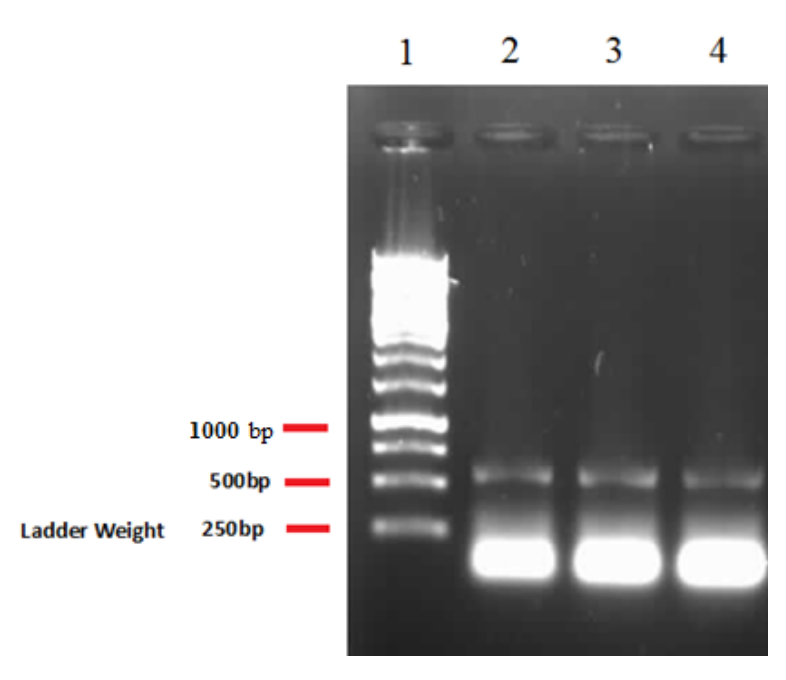

Fig. 1. Results of Nested PCR product. DNA extracted from the blood samples of the ocular toxsoplasmosis patients.

No. 1: Ladder weight (1kb ), No. 2-4: positive samples with 529bp.

demonstrated, in the ocular toxoplasmosis group in the first (second step) and the second follow-up (third step), the antibody titers of IgG became higher or remained unchanged; however, the patients used prescribed medications.

PCR products on $12 \%$ agarose gel showed that band of $529 \mathrm{bp}$ belonged to T. gondii B1 gene (Fig. 1). All PCRpositive samples of patients had positive anti- $T$. gondii IgM or IgG antibodies or both.

\section{Discussion}

The present study demonstrated that clinical diagnostic methods are the gold standard in identifying ocular toxoplasmosis; however, the ophthalmologic signs of $T$. gondii may be mimicked by other infections, or the symptoms may be atypical. Consequently, the possibility of false diagnosis should not be neglected.

To diagnose ocular toxoplasmosis more accurately and control the infection, other new diagnostic approaches, such as serological and molecular methods that are universally employed, should also be considered. These approaches should be used for those patients who are prone to infections (autoimmune disorders; some viruses, especially immunodeficiency virus; organ transplantation; gestation; and some medications). Antibody titers against human $T$. gondii in northern and southern areas of Iran are almost 55\% and 50\%, respectively (5). However, a variety of less common atypical symptoms may be unfamiliar to clinicians and may cause a delay in both diagnosis and treatment, leading to a more severe form of the disease and complete blindness.

Virtually consistent with this study, in a 2-year prospective study of the patients with ocular toxoplasmosis symptoms, the relative diagnostic sensitivity and specificity of PCR, ELISA, and immunoblotting were compared. The results of this study indicated that combining PCR and ELISA for the diagnosis of toxoplasmic chorioretinitis has more precision. Performing PCR on DNA of $T$. gondii and performing both ELISA and immunoblotting to detect the production of local specific antibodies confirmed the toxoplasmic origin of retinal lesions in almost $83 \%$ of patients. Immunoblotting sensitivity in this series was less than ELISA; nevertheless, it may be definite when the blood-retina barrier is impaired. The relative sensitivity and specificity of the aforementioned techniques were $100 \%$ for PCR and $89 \%$ for ELISA and immunoblotting (19).

Almost similar to the present study, in most laboratories, PCR is preferred to other deficient techniques and has consistently been used to detect T. gondii DNA in different biological samples, especially in cases of immunosuppressed and immune-impaired individuals (7, 20, 21). The diagnostic value of PCR to detect $T$. gondii in blood samples has been evaluated from both immunecompetent and compromised patients (12). The serological follow-up demonstrated an increase in toxoplasma-IgG, negative toxoplasma-IgM, and constantly rising IgG, indicating a reactivation before the second transplantation. Donors were seronegative about toxoplasma. Performing PCR on the blood and the liver of the recipient demonstrated toxoplasma-DNA before death (22). Applying the combination of serological tests, culture-based tests, and PCR technique for toxoplasmosis patients results in high diagnostic performance at large (22). Employing laboratory methods, this study demonstrated that out of 71 patients diagnosed with ocular toxoplasmosis in clinical evaluations, $52(73.2 \%)$ had the disease and $18(26.8 \%)$ suffered from other ocular diseases. Although the clinical approach is considered as the gold standard of diagnostic methods, the ophthalmologic signs of $T$. gondii may be mimicked by other infections or the symptoms can be atypical. As a consequence, clinical diagnostic methods may be combined by laboratory approaches for definite and accurate diagnosis of ocular toxoplasmosis. In addition, in this study, PCR showed a significantly higher specificity and sensitivity and a relative diagnostic sensitivity compared to serological evaluation.

Due to the absence of accurate and modern techniques in detection of toxoplasmosis in laboratories, it seems that new diagnosis techniques should be developed. This study aimed to propose some reliable, high-sensitive, and highspecific tests for a correct diagnosis of ocular toxoplasmosis in Iran to prevent unwanted drawbacks and irrecoverable consequences.

\section{Conclusion}

This study benefited from laboratory testing as well as clinical evaluation to reach a reliable and accurate diagnosis of toxoplasmosis. This study also increased the chance of diagnosing $T$. gondii accurately from other mimicked ophthalmologic infections or infections with atypical symptoms. However, this study had some limitations, including the absence of a similar study in different regions of Iran. To deal with this limitation, it is favorable to test ocular liquids, such as aqueous humor, for sampling.

\section{Conflict of Interests}

The authors declare that they have no competing interests. 


\section{References}

1. Commodaro AG, Belfort RN, Rizzo LV, Muccioli C, Silveira C, Burnier Jr MN, et al. Ocular toxoplasmosis: an update and review of the literature. Mem. Inst. Oswaldo Cruz. 2009;104(2):345-50.

2. Montoya JG, Parmley S, Liesenfeld O, Jaffe GJ, Remington JS. Use of the polymerase chain reaction for diagnosis of ocular toxoplasmosis. Ophthalmology. 1999;106(8):1554-63.

3. Araujo FG, Remington JS. Antigenemia in recently acquired acute toxoplasmosis. J. Infect. Dis. 1980;141(2):144-50.

4. Tenter AM, Heckeroth AR, Weiss LM. Toxoplasma gondii: from animals to humans. Int. J. Parasitol. 2000;30(12-13):1217-58.

5. Daryani A, Sarvi S, Aarabi M, Mizani A, Ahmadpour E, Shokri A, et al. Seroprevalence of Toxoplasma gondii in the Iranian general population: a systematic review and meta-analysis. Acta Trop. 2014;137:185-94.

6. Fleck DG, Kwantes W. The laboratory diagnosis of toxoplasmosis: HM Stationery Office; 1980.

7. Savva D, Morris J, Johnson J, Holliman R. Polymerase chain reaction for detection of Toxoplasma gondii. J Clin Microbiol. 1990;32(1):2531 .

8. Jenum P, HOLBERG-PETERSEN M, Melby K, STRAY-PEDERSEN B. Diagnosis of congenital Toxoplasma gondii infection by polymerase chain reaction (PCR) on amniotic fluid samples. Apmis. 1998;106(7-12):680-6.

9. Cingolani A, De Luca A, Ammassari A, Murri R, Linzalone A, Grillo $\mathrm{R}$, et al. PCR detection of Toxoplasma gondii DNA in CSF for the differential diagnosis of AIDS-related focal brain lesions. J Med Microbiol. 1996;45(6):472-6.

10. Held T, Krüger D, Switala A, Beyer J, Kingreen D, Busemann C, et al. Diagnosis of toxoplasmosis in bone marrow transplant recipients: comparison of PCR-based results and immunohistochemistry. Bone Marrow Transplant. 2000;25(12):1257.

11. Mahalakshmi B, Therese KL, Madhavan H, Biswas J. Diagnostic value of specific local antibody production and nucleic acid amplification technique-nested polymerase chain reaction (nPCR) in clinically suspected ocular toxoplasmosis. Ocul Immunol Inflamm. 2006;14(2):105-12.

12. Kompalic-Cristo A, Frotta C, Suárez-Mutis M, Fernandes O, Britto C. Evaluation of a real-time PCR assay based on the repetitive B1 gene for the detection of Toxoplasma gondii in human peripheral blood. Parasitol Res. 2007;101(3):619-25.

13. Meganathan P, Singh S, Ling LY, Singh J, Subrayan V, Nissapatorn V. Detection of Toxoplasma gondii DNA by PCR following microwave treatment of serum and whole blood. Southeast Asian J Trop Med. 2010;41(2):265.

14. Lappalainen M, Hedman K. Serodiagnosis of toxoplasmosis. The impact of measurement of $\mathrm{IgG}$ avidity. Ann Ist Super Sanita. 2004;40(1):81-8.

15. Van Knapen F, Panggabean S. Detection of circulating antigen during acute infections with Toxoplasma gondii by enzyme-linked immunosorbent assay. J Clin Microbiol. 1977;6(6):545-7.

16. Bou G, Figueroa MS, Martí-Belda P, Navas E, Guerrero A. Value of PCR for detection of Toxoplasma gondii in aqueous humor and blood samples from immunocompetent patients with ocular toxoplasmosis. J Clin Microbiol. 1999;37(11):3465-8.

17. Bialvaei AZ, Kafil HS, Asgharzadeh M, Aghazadeh M, Yousefi M. CTX-M extended-spectrum $\beta$-lactamase-producing Klebsiella spp, Salmonella spp, Shigella spp and Escherichia coli isolates in Iranian hospitals. Braz J Microbiol. 2016;47(3):706-11.

18. Wiengcharoen JT, Chiabchalard R, Sukthana Y. PCR technique for detecting Toxoplasma gondii in animal amniotic fluid. Southeast Asian J Trop Med Public Health. 2004;35(4):792-5.

19. Villard O, Filisetti D, Roch-Deries F, Garweg J, Flament J, Candolfi E. Comparison of enzyme-linked immunosorbent assay, immunoblotting, and PCR for diagnosis of toxoplasmic chorioretinitis. J Clin Microbiol. 2003;41(8):3537-41.

20. Čermáková Z, Ryšková O, Plíšková L. Polymerase chain reaction for detection ofToxoplasma gondii in human biological samples. Folia Microbiol. 2005;50(4):341.

21. Tonkal A. PCR versus ELISA in diagnosis of human toxoplasmosis in Jeddah, Saudi Arabia. J Egypt Soc Parasitol. 2008;38(3):707-14.

22. Rostami MN, Eskandari E, Garoosi Z, Mohajeri N, Rezaian M, Keshavarz H. Serological Study of Toxoplasma gondii infection using
IFA method in renal transplant recipients. Iran $\mathrm{J}$ Parasitol. 2006;1(1):31-9. 